\title{
Active Watersheds: Combining 3D Watershed Segmentation and Active Contours to Extract Abdominal Organs from MR Images
}

\author{
R.J. Lapeer ${ }^{1}$, A.C. Tan $^{2}$, and R. Aldridge ${ }^{1}$ \\ ${ }^{1}$ School of Information Systems, University of East Anglia, Norwich NR4 7TJ, UK \\ ${ }^{2}$ Department of Medical Physics and Bioengineering, University College London
}

(UCL), London WC1E 6JA, UK

\begin{abstract}
The three-dimensional segmentation of regions of interest in medical images, be it a $2 \mathrm{D}$ slice by slice based approach or directly across the $3 \mathrm{D}$ dataset, has numerous applications for the medical professional. These applications may involve something as simple as visualisation up to more critical tasks such as volume estimation, tissue quantification and classification, the detection of abnormalities and more. In this paper we describe a method which aims to combine two of the more popular segmentation techniques: the watershed segmentation and the active contour segmentation. Watershed segmentation provides unique boundaries for a particular image or series of images but does not easily allow for the discrete nature of the image and the image noise. Active contours or snakes do possess this generalisation or smoothing property but are difficult to initialise and usually require to be close to the boundary of interest to converge. We present a hybrid approach by segmenting a region of interest (ROI) using a 3D marker-based watershed algorithm. The resulting ROI's boundaries are then converted into a contour, using a contour following algorithm which is explained during the course of the paper. Once the contours are determined, different parameter settings of internal/external forces allow the expert user to adapt the initial segmentation. The approach thus yields a fast initial segmentation from the watershed algorithm and allows fine-tuning using active contours. Results of the technique are illustrated on 3D colon, kidney and liver segmentations from MRI datasets.
\end{abstract}

\section{Introduction}

Segmentation of images, and more specifically medical images, is a field which has sparked exciting research throughout the last few years. However it has also caused as much controversy due to divided opinions on the benefits and drawbacks of particular segmentation techniques for particular tasks. Three standard groups of segmentation are distinguished typically as: region-based, edge-based and classification-based [3]. Most segmentation techniques in medical imaging focus on a well-defined task, for example a certain structure or tissue of a particular image modality; often this is even further specialised for a certain objective, 
for example volume estimation and quantification or diagnosis of abnormal tissues. The aim of the research, as presented in this paper, is to create a more general, hierarchical segmentation methodology which allows a trade-off between speed and accuracy. At this stage, the initial segmentation uses a 3D markerbased watershed algorithm which gives fast results but not necessarily with the expected accuracy as little consideration is given to image noise and discretisation. To overcome this difficulty, the resulting watersheds are converted into active contours using a contour following algorithm. Active contours possess the smoothing properties which watershed lack, however the latter solves the initialisation and connectivity problems of the former.

In the next sections we discuss the established watershed and active contour models that we have used and elaborate on the contour following algorithm. The final section shows, and discusses, results on colon, liver and kidney segmentations. Also, in the final section, we discuss the technique as it currently stands together with possible extensions to create a more versatile and intelligent' system which automatically selects appropriate techniques for different medical image segmentation tasks.

\section{Methodology}

\subsection{D Marker-Based Watershed Segmentation}

Watershed segmentation is a morphological region-based segmentation technique. Although first applied to grey-scale images as early as 1979 by Buecher and Lantuéjoul [1] the technique only became popular in the early 90's in a variety of fields in need of automatic image segmentation. This was as a result of the publication of a watershed algorithm by Vincent and Soille [10] and the rapid growth of the computational power of workstations. This algorithm uses a 2D grey-scale image as an input (which can be extended to a full 3D dataset), typically pre-processed to produce a smoothed gradient image. For ease of understanding the watershed principle, let us momentarily forget the 3D extension and assume a 2D image and its different grey-levels represented as a relief. Imagine this relief (looking like a landscape with hills and troughs) to be flooded by water. Water will settle first in a basin - typically called a catchment basin and raise until it meets one or more neighbouring catchment basins. To avoid water spilling from one basin to the other we construct a dam of single-pixel width, which is higher than the highest peak $(\mathrm{s})$ in the image relief. When the water has reached the highest peak(s) in the image, the resulting dams form the watershed lines which separate the basins. As the catchment basins represent different regions in the image, we arrive at a mosaic-like segmentation of that image. The problem with the standard approach is that depending on the noise in the image, too many regions (possibly belonging together) are separated, which means the image is over-segmented (see Fig:1(b)). Techniques to counteract over-segmentation are mainly based on morphological pre-processing using automatically generated internal and external markers - an overview of 


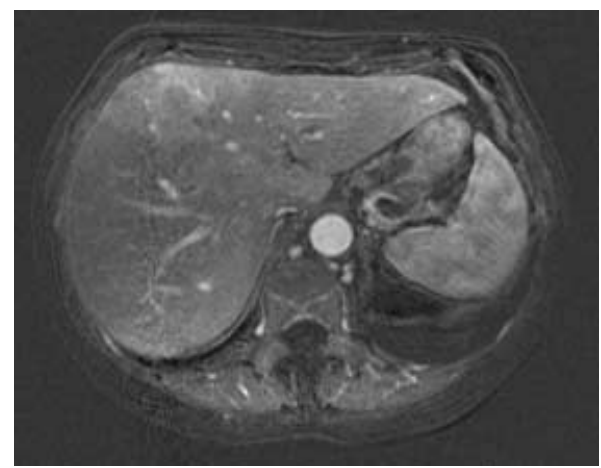

(a)

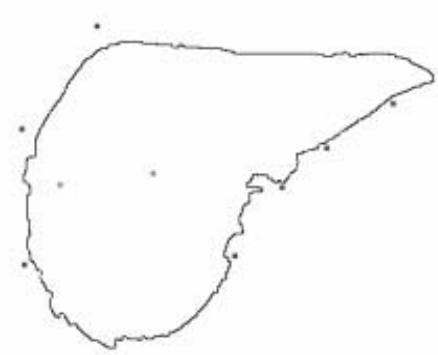

(c)

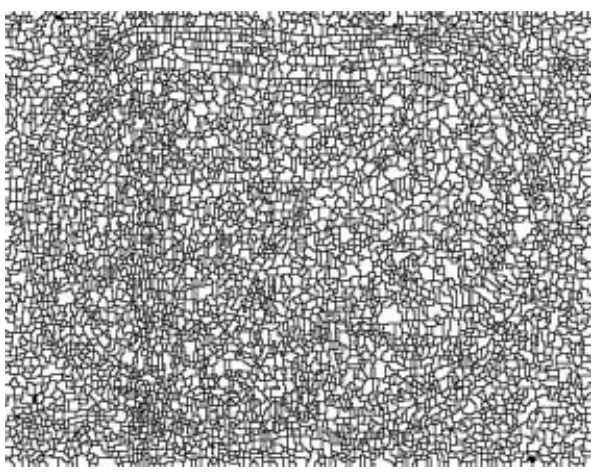

(b)

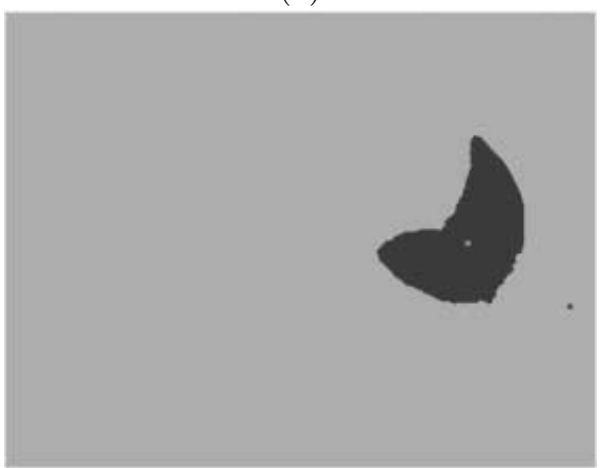

(d)

Fig. 1. The original MRI image (a), the over-segmented image using the standard Vincent and Soille algorithm (b), and avoiding this problem using manually placed internal (light) and external (dark) markers for liver segmentation showing the watershed boundary (c) and spleen segmentation showing the internal and external catchment basins $(\mathrm{d})$.

such techniques can be found in 4]. Although the techniques have proved successful for certain images (e.g histological images), they often require several trials using different pre-processing combinations and, even then, are not always capable of producing unambiguous segmentation results. Therefore, we opted for a semi-interactive approach, using manually placed internal and external markers. The method requires the user, typically a medical expert, to place one or more internal markers in the ROI and one or more external markers in the rest of the image (see Fig 1 (c) and (d)). If the final segmentation is unsuccessful more markers can be added or removed to yield a satisfactory result. This approach also guarantees a segmentation into two distinct regions, i.e. the region of interest (ROI) and the background.

The algorithm can be extended easily from $2 \mathrm{D}$ to $3 \mathrm{D}$ as the principle of the image 'flooding' is based on the ordering of pixels in each slot of a cumulative distribution of grey-values of the image (typically 0-255 for 8 bit); each grey-level 
containing an array of all pixels having that grey-value. Thus, any grey-scale image or dataset of finite dimension can be processed using the same algorithm provided the gradient calculations are adapted.

\subsection{A Contour Following Algorithm to Convert the Watershed into an Active Contour}

The watershed boundaries are unfortunately not perfect; they may consist of a boundary of several pixels wide, rather than one, and may contain gaps and glyphs (e.g. pixels 10N-10Q in Fig,2). We have implemented a tree-based contour following algorithm using 8-connectednes to create a closed contour with edges of neighbouring pixels:

step 1 Search from the top downwards, the first leftmost pixel in the binary bitmap - e.g. pixel D8 in Fig 2

Make this pixel the initial and current pixel.

step 2 From the current pixel which is at level 0 in the tree, process in clockwise direction (just a convention ...).

Find the next boundary pixel(s) in the 8-connected neighbourhood whilst ignoring pixels in the invalid direction.

IF only one valid boundary pixel exist make this pixel the common pixel. Go to step 4.

IF no valid boundary pixels exist (which means there is a gap) expand nonboundary pixels in the right direction. Keep expanding the tree breadthfirst until a level is reached containing a common single pixel - e.g. in Fig 2 pixel G12, will expand in valid non-boundary pixels G11,G13,H11-13, which will eventually all end in I14.

IF multiple candidate boundary pixels exist, expand until the tree reaches a level with a common single pixel - e.g. pixel P11 will yield three valid boundary pixels: O10,P10 and Q10. They will eventually all yield pixel P9 at the next level. Pixel N10, spawned by O10 would eventually 'die' as it has no valid successors (as only at level 0 , non-boundary pixels are spawned).

step 3 From the common pixel at the bottom of the tree, find the 'least-effort' path. The latter implies a path with average curvature.

step 4 Connect the current pixel to the common pixel using this path and make the common pixel the current pixel.

step 5 Go back to step 2 until the current pixel is the initial pixel.

Notes:

- A queue structure is required to expand the tree in breadth-first fashion.

- A previous current pixel (that is a pixel which is now part of the contour) becomes an invalid pixel when expanding its successors.

- Previously visited pixels which did not become part of the contour because they failed the 'least-effort' path test, become invalid too.

- When expanding the tree, valid pixels already in a higher level in the tree are not considered (which avoids a 'shortest path' test). 


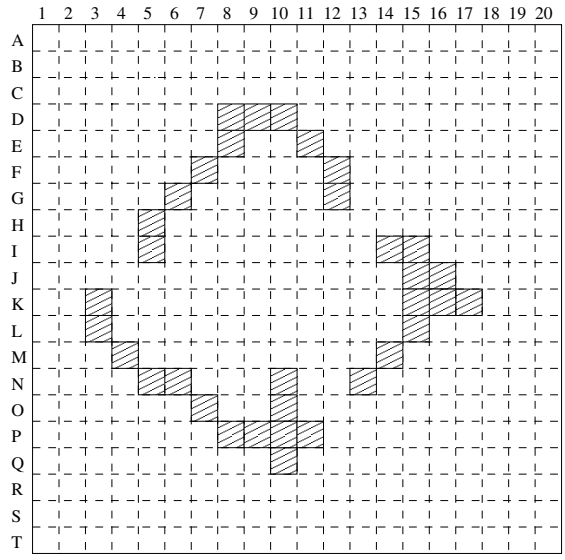

Fig. 2. A model boundary of a watershed containing gaps, multi-pixel width and glyphs.

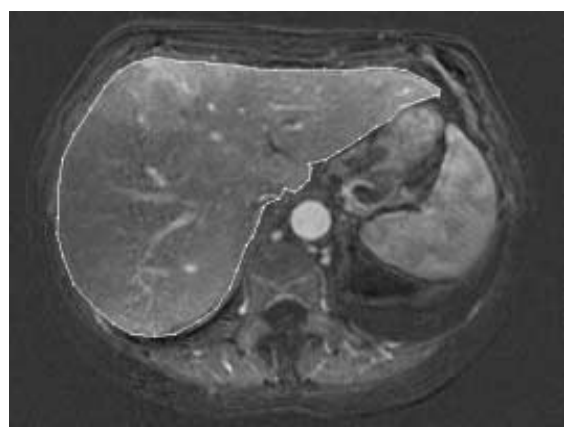

Fig. 3. The segmented liver with active contour turned watershed.

- The final contour can be post-processed to turn edges with the same slope into a single edge.

\subsection{Active Contours}

Active contours have become popular as a model for image segmentation mainly by Kass, Witkin and Terzopoulos [5]. They have since then been subjected to several optimisation models and 3D extensions of which a fairly recent update is given in [11. In this paper, we used the standard simplified active contour model (without inertial term):

$$
\gamma \frac{\partial \mathbf{X}}{\partial t}=F_{\text {int }}(\mathbf{X})+F_{\text {ext }}(\mathbf{X})
$$

with $\mathbf{X}(s)$, the position vector of a contour element ( $s$ being the curve parameter), internal (smoothing) forces, $F_{\text {int }}(\mathbf{X})$, and external forces (Gaussian potential force derived from the image), $F_{\text {ext }}(\mathbf{X})$. The factor $\gamma$ is a damping coefficient. A finite difference scheme can be used for numerical implementation.

\section{Preliminary Segmentation Results}

Fig.3 shows a processed active contour, initially derived from the watershed, as shown in Fig.1(c), using the contour following algorithm. Fig.4(a) shows the result of a $3 \mathrm{D}$ watershed segmentation. Fig.4(b) shows the result after application of active contours on the watershed contours in each slice of the MR dataset. The dataset is of size $512 \times 512 \times 56 \times 16$ bit. Four internal markers 


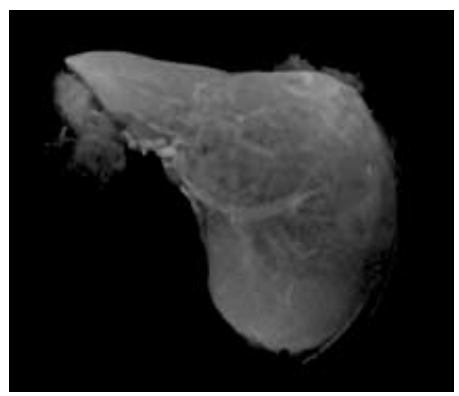

(a)

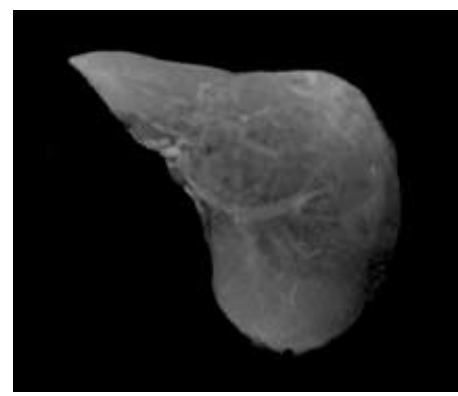

(b)

Fig. 4. 3D segmentation of liver with original texture-map in the segmented region: initial segmentation using a 3D watershed algorithm (a); smoothed segmentation after active contour fitting (b). MRI data of $512 \times 512 \times 56 \times 16$ bit was used.

and twenty external markers were needed for the watershed segmentation. The processing time was about 3 minutes on an SGI Onyx InfiniteReality. Fig[5(a) shows two kidneys segmented from a $256 \times 256 \times 128 \times 8$ bit MR dataset. Only two internal and three external markers in just one slice (out of 128) was needed. No active contour smoothing was applied. Fig.5.(b) and (c) show segmentation of the colon from the same dataset. Only one internal and three external markers were needed, no smoothing. Note that the original texture was mapped into the segmented regions to visualise the internal structures.

\section{Discussion}

The example in Fig 4(a) and (b) typically shows the shortcomings of the watershed algorithm. Even with the application of a sufficient number of markers, the $3 \mathrm{D}$ segmentation of the liver remains prone to 'noisy' regions, caused by the internal ROI's catchment basin 'leaking' into inherently external regions. Adding external markers to the boundaries where these regions merge avoids this problem but usually results in under-segmentation. This means that parts of the (internal) ROI have now become part of the (external) background. The case of liver segmentation is a difficult task because of its inhomogeneous and lobelike structure. Here, the watershed method by itself fails to produce a noise-free segmentation. The subsequent use of active contours smoothens out the noisy (background) regions. The results from segmenting the colon and kidneys can be further smoothed using active contours, however the watershed segmentation appears to cope very well. A good indicator to its success is the number of internal and external markers needed to produce a relatively noise-free segmentation. For the colon segmentation, which can be used typically for virtual colonoscopy, we also tested a seed-growing approach. We concluded that the watershed algorithm outperforms this method as it copes better with connectivity problems due to its more rigorous boundary criteria. 


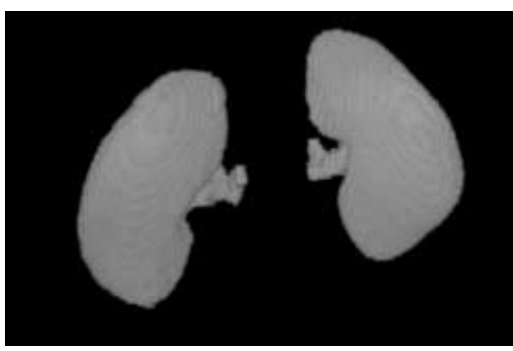

(a)

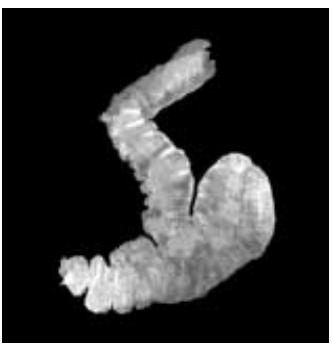

(b)

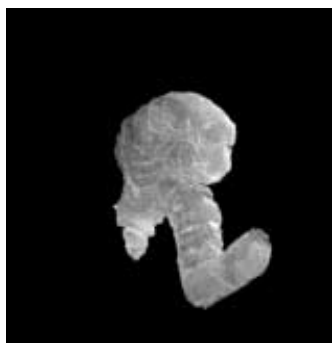

(c)

Fig. 5. Segmentation (3D with texture map using original data) of kidneys (a) and two views, (b) and (c), of a segmented colon from a $256 \times 256 \times 128 \times 8$ bit MRI dataset.

Although we know that each individual method is capable of successful segmentation of particular organs for particular modalities, the sum of the two should not normally be worse than each individual method. Care has to be taken however to avoid that one method renders the results of the other method void. For example, the watershed may settle around an undesirable boundary, which subsequently used by the active contour as its initial state, may render the latter's segmentation incorrect or more cumbersome as compared to using the active contour without the watershed. Also the active contour segmentation may destroy the original watershed segmentation for the same reason.

Currently the watershed uses $3 \mathrm{D}$ gradient information and the active contours use a 2D (within-slice) gradient. To allow for this discrepancy, the watershed could be used in a 2D slice-by-slice mode (which still produces a 3D segmentation) so both methods use $2 \mathrm{D}$ gradient information. A better alternative is to use active surfaces instead of active contours. The 3D watershed regions are iso-surfaces which can be used as an initial model to an adaptive deformable triangulated surface [8] after triangulation (e.g. using a marching-cubes technique [7]). This could be a better alternative to the use of multi-resolution volume pyramids in [8]. As this extension would require more processing resources, the current immersion-based water shed algorithm can be speeded up using a rainfall algorithm 9 .

Using automatically generated markers as an alternative to manually placed markers can be further investigated using morphological filters [2], pixel statistics based methods or boundary extraction using transparency functions [6].

To further test the method, different image modalities and structures have to be investigated. For example, deformable models have been widely used for brain segmentation because of the complex geometry of brain structures. However, in [2] it is shown that marker-based watershed segmentation is also capable of successfully segmenting the ventricles, hippocampus, corpus callosum and cerebellum. It would be interesting to evaluate the combined watershed/active contour approach to the segmentatio $n$ of these structures.

Finally, the tool could be extended to incorporate some form of artificial intelligence (AI) by selecting the appropriate methods for certain tasks. It was already 
pointed out that the number of markers needed for the watershed segmentation proves to be a good indicator of its potential success. Quantitative information like this together with prior (expert) information could then be used to make automatic decisions for optimal segmentation.

\section{Conclusion}

The combination of watershed segmentation and active contours proves to be a potentially promising tool for medical image segmentation. We currently have successfully tested the method on a limited number of MR datasets for the segmentation of abdominal organs. More tests are anticipated to further test its potential on different structures using different imaging modalities.

\section{References}

1. S. Buecher and C. Lantuéjoul. Use of watershed in contour detection. In Proc. Int. Workshop Image Processing, Real-Time Edge and Motion Detection/Estimation, Rennes, France, pages 17-21, September 1979.

2. G. Bueno, O. Musse, F. Heitz, and J.P Armspach. Three-dimensional segmentation of anatomical structures in MR images on large databases. Magnetic Resonance Imaging, 19:73-78, 2001.

3. B.M. Dawant and A.P. Zijdenbos. Chapter 2: Image segmentation. In J.M Fitzpatrick and M. Sonka, editors, Handbook of Medical Imaging. Vol.2 Medical Image Processing and Analysis, pages 71-127. SPIE, London, June 2000.

4. J. Goutsias and S. Batman. Chapter 4: Morphological methods for biomedical image analysis. In J.M Fitzpatrick and M. Sonka, editors, Handbook of Medical Imaging. Vol.2 Medical Image Processing and Analysis, pages 175-272. SPIE, London, June 2000.

5. M. Kass, A. Witkin, and D. Terzopoulos. Snakes: active contour models. Int.J.Comp.Vis., 1(4):321-331, 1987.

6. G. Kindlmann and J. Durkin. Semi-automatic generation of transfer functions for direct volume rendering. In Proc. IEEE Symposium on Volume Rendering, October 1998.

7. W.E. Lorensen and H.E. Cline. Marching cubes: a high resolution 3d surface construction algorithm. Computer Graphics, 21(4):163-169, 1987.

8. J-Y. Park, T. McInerney, D. Terzopoulos, and M-H. Kim. A non-self-intersecting adaptive deformable surface for complex boundary extraction from volumetric images. Computers $\& 3$ Graphics, 25:421-440, 2001.

9. P. De Smet and D. De Vleeschauwer. Performance and scalability of a highly optimized rainfalling watershed algorithm. In Proc. of the 1998 Int.Conf. on Imaging Science, Systems and Technology, CCIST'98 - Las Vegas, pages 266-273, July 1998.

10. L. Vincent and P. Soille. Watersheds in digital spaces: An efficient algorithm based on immersion simulations. IEEE Trans. Patt.Anal.Mach.Int., 13(6):583-598, June 1991.

11. C. Xu, D.L. Pham, and J.L. Prince. Chapter 3: Image segmentation using deformable models. In J.M Fitzpatrick and M. Sonka, editors, Handbook of Medical Imaging. Vol.2 Medical Image Processing and Analysis, pages 175-272. SPIE, London, June 2000. 